\title{
LncRNA MEG3 Induces Endothelial Differentiation of Adipose-derived Stem Cells by Targeting MiR- 145-5p/KLF4
}

\section{Hailong Zhang}

First Affiliated Hospital of Heilongjiang University Traditional Chinese Medicine

\section{Gang Liu}

Second Affiliated Hospital of Traditional Chinese Medicine

\section{Xu Mao}

Heilongjiang University of Chinese Medicine

Lei Yang

Heilongjiang Academy of Traditonal Chinese Medicine

\section{Bingyu Wang}

Heilongjiang Academy of Traditional Chinese Medicine

Xingxing Yuan ( $\nabla$ jackie198711@163.com )

Heilongjiang Academy of Traditional Chinese Medicine https://orcid.org/0000-0002-9894-4127

\section{Research Article}

Keywords: adipose-derived stem cells, long non-coding RNA maternally expressed 3, microRNA-145-5p, Kruppel like factor 4, endothelial differentiation

Posted Date: February 21st, 2022

DOI: https://doi.org/10.21203/rs.3.rs-1276866/v1

License: (9) This work is licensed under a Creative Commons Attribution 4.0 International License. Read Full License 


\section{Abstract}

Background: The present study aimed to investigate the mechanisms through which long non-coding RNA (IncRNA) maternally expressed 3 (MEG3) affected the endothelial differentiation of adipose-derived stem cells (ADSCs).

Material and Methods: ADSCs were isolated and identified by specific surface marker detection. The effects of IncRNA MEG3 on endothelial differentiation of ADSCs were also detected via quantitative PCR, western blotting, immunofluorescence and Matrigel angiogenesis assays. In addition, using target gene prediction tools and luciferase reporter assays, the downstream target gene was demonstrated.

Results: LncRNA MEG3 targeted and reduced the expression levels of microRNA-145-5p (miR-145-5p), which upregulated the expression levels of Krüppel like factor 4 (KLF4), promoting endothelial differentiation of ADSCs.

Conclusion: LncRNA MEG3 induced endothelial differentiation of ADSCs by targeting miR-145-5p/KLF4, which may provide novel insights to illustrate the mechanism of endothelial differentiation of ADSCs.

\section{Introduction}

Wound repair and regeneration are some of the most complex biological processes. However, dysregulation of cellular and molecular signals may lead to excessive wound healing, including hypertrophic scar formation or inadequate healing under conditions such as ischemia, diabetes, infection and radiation exposure, which further results in the loss of body parts, decreased quality of life and even mortality due to wound-related complications ${ }^{1}$. The latest developments in this emerging field of regenerative medicine may make it possible to repair tissue damage and eventually replace organs. Therefore, identifying a type of seed cell, which are easy to obtain and expand in vitro, has become the focus of regenerative medicine and tissue engineering research.

Stem cells, due to their high proliferative ability and multi-directional differentiation potential, are the best choice for seed cells in regenerative medicine ${ }^{2}$. Among adult stem cells, adipose-derived stem cells (ADSCs) are pluripotent stem cells originating from the mesoderm, and are also called adipose-derived stromal cells. These cells theoretically have the potential to differentiate into endothelium, epithelial cell or fibroblast lineages ${ }^{3-5}$. Compared with obtaining bone marrow mesenchymal stem cells, obtaining ADSCs is more convenient, and has a less detrimental impact on the body ${ }^{6}$. Since it is easy to culture ADSCs in vitro at a large scale and to induce their differentiation, ADSCs have been demonstrated to have the potential of wound healing. On the one hand, endothelial differentiation of ADSCs can promote angiogenesis in ischemic conditions via secretion of angiogenic and anti-apoptotic factors, such as VEGF

7. On the other hand, it can also increase the thickness and collagen content of granulation tissue, and improve the cosmetic appearance of resultant scars, thereby promoting wound healing even under unfavorable conditions ${ }^{8}$. However, studies on the differentiation of ADSCs into endothelial cells are 
limited, thus restricting the development of functional methods for endothelial differentiation. Therefore, it is of great importance to explore the underlying mechanism of endothelial differentiation of ADSCs.

Long non-coding RNAs (IncRNAs) can regulate a large number of genes and serve an important role in the differentiation of a number of adult stem cells ${ }^{9}$. Among these, maternally expressed 3 (MEG3), which has multiple regulatory functions in various normal and pathological cells, has become a research hotspot in the IncRNA field. In the early stages of research, the function of IncRNA MEG3 was mostly identified in tumor cells ${ }^{10-12}$. With in-depth study, researchers have revealed that MEG3 is also involved in regulating biological processes ${ }^{13,14}$, such as differentiation of stem cells ${ }^{15,16}$. You et a/ ${ }^{17}$ revealed that IncRNA MEG3 regulated synovium-derived mesenchymal stem cell differentiation by inhibiting tribbles pseudokinase 2 expression. Li et al ${ }^{18}$ reported that MEG3 was involved in the balance of adipogenic and osteogenic differentiation of adipose-derived mesenchymal stem cells, and the mechanism may be via sponging of microRNA (miRNA/miR)-140-5p. However, to the best of our knowledge, the role of MEG3 in endothelial cell differentiation of adipose stem cells remains unknown.

The present study aimed to investigate the role of MEG3 in endothelial differentiation of ADSCs. It revealed the activation effect of MEG3 on endothelial differentiation of ADSCs, and its downstream miR145-5p/Krüppel like factor 4 (KLF4) axis, which mediated the function of MEG3. The present study may provide proof-of-principle evidence of the mechanism via which MEG3 regulates endothelial differentiation of ADSCs.

\section{Materials And Methods}

\section{Animals}

20 C57BL/ 6 male wild-type mice (age, $~ 6$ weeks) were provided by Beijing Vital River Laboratory Animal Technology Co., Ltd. After 1 week of diet adaptation, mice at the age of 8 weeks were fed on a high-fat diet. During this period, the fresh feed was changed every 3 days. The weight of the mice was measured every 2 weeks and the high-fat diet was given to the model animals for 6 months. All operations were approved by the Ethics Research Committee of Heilongjiang Academy of Traditional Chinese Medicine. All mice were maintained in ventilated cages with a 12-h light/dark cycle and free access to food and water at $26^{\circ} \mathrm{C}$ and stable atmospheric pressure. Mice were euthanized via an peritoneal injection of $1 \%$ sodium pentobarbital $(120 \mathrm{mg} / \mathrm{kg})$, and the procedures were consistent with the American Veterinary Medical Association Guidelines for the Euthanasia.

Isolation and characterization of ADSCs: Healthy adult male C57BL/6 mice weighing 30-35 g were included in the present study. Mice were anesthetized with $1 \%$ sodium pentobarbital (dose, $50 \mathrm{mg} / \mathrm{kg}$ ), and the periaortic adipose tissues surrounding the thoracic aorta (from the aortic arch to the aortic hiatus) were pooled for each primary culture. Fresh adipose tissue was digested with $0.075 \%$ type I collagenase and centrifuged at a rotational speed of $800 \times \mathrm{g}$ for $10 \mathrm{~min}$ at room temperature. The supernatant was removed, and tissue cell masses could be collected at the bottom of the tube. After the 
low glucose-DMEM (Gibco, 11885092) containing 10\% FBS (Gibco, 10099141) was replaced, the cell petri dishes inoculated to $100 \mathrm{~mm}$ were cultured at $37^{\circ} \mathrm{C}$ with $5 \% \mathrm{CO}_{2}$. When the cells grew to $>80 \%$ of the culture dish, the culture medium was removed and passaged at the proportion of 1:3. After the third generation, the expression levels of ADSC markers CD44 (cat. no. 553133, 1:200 dilution), CD90 (cat. no. 555596, 1:200 dilution), CD31 (cat. no. 558738, 1:200 dilution) and HLA-DR (major histocompatibility complex, class II antigen-associated) (cat. no. 555318, 1:200 dilution) were detected via flow cytometry. All these antibodies were purchased from BD Biosciences.

Cell treatment: MEG3 short hairpin RNA (shRNA), KLF4 shRNA, miR-145-5p inhibitor and miR-145-5p mimic were purchased from Shanghai GenePharma Co., Ltd. Under the specified infection and treatment conditions, these RNAs have no off-targeted effect, nor do they affect the adherence, shape or activity of ADSCs. In the gene knockdown experiment, shRNA targeting MEG3 (5'-AAG ACC AGT ATG GCG TGG CTC TCTT-3') and KLF4 (5'-CAC CCA CAC TTG TGA CTAT-3') was commercially synthesized and cloned into the pLKO.1 vector (MilliporeSigma). The sequences of miR-145-5p inhibitor and miR-145-5p mimic were synthesized as follows: miR-145-5p mimic, 5' -GUC CAG UUU UCC CAG GAA UCC CU-3'; negative control (NC) mimic, 5' -UUC UCC GAA CGU GUC ACG UTT-3'; miR-145-5p inhibitor, 5'-GGA AUC CCU UAG AUG CUAA -3'; and NC inhibitor, 5'-GAT GGT CTT GCG GTC GTA GAT-3'. For overexpression of MEG3, human full length MEG3 cDNA was subcloned into pcDNA vector (Guangzhou Ribobio Co., Ltd) and pcDNA-3.1 was adopted as control. When the cell density reached $70-80 \%$, plasmids $(2 \mu \mathrm{g} /$ well), and miRNA mimics $\left(50 \mathrm{nM} /\right.$ well) or inhibitor (50 nM/well) were transfected using Lipofectamine ${ }^{\circledR} 3000$ (Thermo Fisher Scientific, Inc.) according to the manufacturer's protocol. Cells were incubated at $37^{\circ} \mathrm{C} / 5 \% \mathrm{CO}_{2}$ and were used for subsequent experiments 36 post-transfection. The transfection efficiency in ADSCs is shown in Fig. S1.

To induce ADSCs to differentiate into vascular endothelial cells, the petri dishes were coated with 5 $\mu \mathrm{g} / \mathrm{cm}^{2}$ fibronectin ( $\mathrm{FN}$; BD Biosciences). $\mathrm{FN}$, as an important part of the extracellular matrix, not only acts as a scaffold for cells but is also an important connecting factor between cells. Subsequently, the third-passage ADSCs were seeded into the FN-coated dishes and incubated with EGM2-MV (Lonza Group, Ltd.) $+50 \mathrm{ng} / \mathrm{ml} \mathrm{VEGF}_{165}$ (PeproTech China). EGM2-MV is an endothelial cell support medium, which contains a variety of cytokines, including recombinant human epidermal growth factor, recombinant human fibroblast-like growth factor and insulin-like growth factor. In addition, VEGF is an important factor in maintaining endothelial differentiation and proliferation.

Flow cytometry analysis: The third-passage ADSCs were digested and centrifuged at a rotational speed of $800 \times \mathrm{g}$ for $10 \mathrm{~min}$ at room temperature. After washing with 0.5\% BSA (Beyotime, ST025)-PBS solution three times, the density of the single cell suspension was adjusted to $1 \times 10^{6} \mathrm{cells} / \mathrm{ml}$. Rat anti-mouse CD44 (cat. no. 553133, 1:200 dilution), CD90 (cat. no. 555596, 1:200 dilution), CD31 (cat. no. 558738, 1:200 dilution) and HLA-DR (major histocompatibility complex, class II antigen-associated) (cat. no. 555318, 1:200 dilution) (FITC label; all BD Biosciences) monoclonal antibodies were added, and fluorescence-labeled non-specific IgG was added to a tube as a homotype control. The cells were 
incubated at $37^{\circ} \mathrm{C}$ for $30 \mathrm{~min}$. PBS was used for washing three times, $1 \%$ paraformaldehyde was used for fixing and then antigen expression was detected using a FACSCalibur flow cytometer (BD Biosciences).

Reverse transcription-quantitative PCR (RT-qPCR): According to the manufacturer's instructions, the RNA of samples was extracted for subsequent experiments. Briefly, total RNA was extracted using TRIzol® reagent (Beijing Solarbio Science \& Technology Co., Ltd.) and finally dissolved in diethyl pyrocarbonate water. Subsequently, cDNA was obtained using a reverse transcription PrimeScript 1 st Strand cDNA synthesis kit (Takara Bio, Inc.). RT-qPCR was performed using SYBR Green Q-PCR mix (Applied Biosystems; Thermo Fisher Scientific, Inc.) on a Roche LightCycler 480 (Roche Diagnostics). Relative gene expression was calculated using the $2^{-\triangle \triangle C q}$ method with GAPDH mRNA as an internal control. The probes were synthesized as follows: GAPDH forward, 5'-GGG AGC CAA AAG GGT CAT-3' and reverse, 5'GAG TCC TTC CAC GAT ACC AA-3'; U6 forward, 5'-CCG CCC GCC GCC AGG CCCC-3' and reverse, 5'-ATA TGG AAC GCT TCA CGA ATT-3'; miR-145-5p forward, 5'-GUC CAG UUU UCC CAG GAA UCC CU-3' and reverse, 5'-TGG TGT CGT GGA GTC G-3'; CD31 forward, 5'-CAC TTC TGA ACT CCA ACA ACG-3' and reverse, 5'-GGA CAC TTG AAC TTC CGTG-3'; von Willebrand factor (vWF) forward, 5'-ATG GTT CTG GAT GTG GCGT-3' and reverse, 5'-TTG CTC CTG TTG AAG TCGG-3'; and Factor VIII forward, 5'-GGA GAG ATG GGA CAC TAA CA-3' and reverse, 5'-ACT GAA CCT GAC CGT ACA GTC ATA TGG ACG ACT GAG GT-3'.

Western blotting: To extract total protein, samples were treated using the subsequent procedure. The culture medium in the petri dish was removed and gently washed with PBS three times to remove residual medium. Subsequently, RIPA lysate containing 1\% PMSF [50 mM Tris (pH 7.4), $150 \mathrm{mM} \mathrm{NaCl}, 1 \%$ TritonX$100,1 \%$ sodium deoxycholate, $0.1 \%$ SDS, $2 \mathrm{mM}$ sodium pyrophosphate] was added for lysis on ice for 30 min. The lysate was centrifuged at $1000 \times \mathrm{g}$ at $4^{\circ} \mathrm{C}$ for $10 \mathrm{~min}$. The supernatant was transferred to a new tube for protein quantification using a BCA assay. Subsequently, $12 \%$ SDS-PAGE was performed, followed by transfer onto a PVDF membrane (MilliporeSigma). After blocking with $5 \%(\mathrm{w} / \mathrm{v})$ non-fat dry milk, the membranes were incubated with primary antibodies against $\beta$-actin (cat. no.ab8226, 1:2,000 dilution), CD31 (cat. no.ab9498, 1:1,000 dilution), vWF (cat. no.174290, 1:1,000 dilution) and Factor VIII (cat. no.ab275376, 1:1,000 dilution). All antibodies were purchased from Abcam Group, Inc. Subsequently, the appropriate HRP-conjugated secondary antibodies (cat. no.SA00001-2, 1:5,000 dilution) were purchased from Proteintech Group, Inc.. The protein bands were detected using the chemiluminescence procedure (Pierce; Thermo Fisher Scientific, Inc.) on a Tanon 5200 Imaging system (Tanon Science and Technology Co., Ltd.).

Immunofluorescence: The cells were inoculated into a petri dish with pre-treated glass slides. When the density reached $70-80 \%$, slides of cells were fixed with $4 \%$ paraformaldehyde and permeated with $0.5 \%$ Triton. Subsequently, the cells were washed with PBS three times and blocked with $3 \%(w / v)$ BSA (Beyotime, ST025) at $4^{\circ} \mathrm{C}$ overnight. Afterwards, the cell slides were incubated with primary antibodies against CD31 (cat.no.11265-1-AP, 1:500 dilution, proteintech) at $4^{\circ} \mathrm{C}$ overnight. Following incubation with FITC-conjugated secondary antibodies (cat.no.SA00013-2, 1:1,000 dilution, proteintech) at room temperature for $1 \mathrm{~h}$, the slides were observed under a fluorescence microscope (IX73; Olympus Corporation). 


\section{Matrigel angiogenesis assay}

The Matrigel angiogenesis assay was performed using an in vitro angiogenesis kit (Abcam). The electrolyte gel solution $(50 \mu \mathrm{l})$ was added to the precooled 96 -well plate and solidified at $37^{\circ} \mathrm{C}$ for $20 \mathrm{~min}$. ADSCs $\left(2 \times 10^{4}\right)$ were seeded in DMEM (Gibco, 11885092) containing 10\% FBS and cultured for $24 \mathrm{~h}$. The image was captured using a DMI 6000B microscope (Leica Microsystems, Inc.).

Luciferase reporter gene assay: Using target gene prediction online software (TargetScanHuman v7.2, http://www.targetscan.org/vert_72/), it was preliminarily predicted that miR-145-5p was the target gene of MEG3, and KLF4 was downtarget of miR-145-5p. The 3' untranslated regions (3'-UTRs; wild type) and the corresponding mutant 3 '-UTRs were amplified by PCR. The obtained target fragments were cloned into the pLVX reporter vector. 293T cells (CL-0005, Procell) were inoculated into a 24-well plate at a concentration of $1 \times 10^{5}$ cells $/ \mathrm{cm}^{2}$. When the cell density reached $70-80 \%$, reporter gene plasmids and miR145-5p were co-transfected into 293T cells using Lipofectamine ${ }^{\circledR} 2000$ (Thermo Fisher Scientific, Inc.). The cells were collected at $48 \mathrm{~h}$ after transfection and the luciferase activity was detected.

\section{Statistical analysis}

For each experiment, each group of experiments was repeated three times independently. Data are presented as the mean \pm SD. Independent-samples t-tests assuming equal variance and Two-way-ANOVA assay were used for statistical analysis, and the SPSS17.0 software (SPSS, Inc.) was used. P $<0.05$ was considered to indicate a statistically significant difference.

\section{Results}

\section{Characterization of ADSCs}

To identify the isolated cells, the third generation of ADSCs was detected using the cell surface antigen via flow cytometry. The results demonstrated that anti-CD44 and anti-CD90 exhibited positive signals (Fig. 1A), indicating that ADSCs had a common surface marker of mesenchymal stem cells. Additionally, anti-CD31 and anti-HLA-DR exhibited negative signals (Fig. 1B), thus excluding the contamination of endothelial cells and hematopoietic lines.

\section{ADSCs have the ability to differentiate into vascular endothelial cells}

To confirm that the ADSCs have the ability to differentiate into vascular endothelial cells, the third generation of ADSCs was induced as described previously. Subsequently, samples were collected at different time points ( 0,7 and 15 days), and the changes of vascular endothelial cell-specific marker mRNA levels were detected. The results of fluorescence qPCR revealed that the mRNA expression levels of CD31, vWF and Factor VIII gradually increased (7 days, $P<0.01 ; 15$ days, $P<0.01$; Fig. $2 A$ ). In addition, after third-generation ADSCs were induced for the indicated number of days, the protein expression levels of CD31, vWF and Factor VIII gradually increased in a time-dependent manner (7 days, $P<0.05 ; 15$ days, 
$\mathrm{P}<0.01$; Fig. 2B). To further evaluate the differentiation of ADSCs, the present study examined the CD31 expression in these differentiated cells and revealed that the expression levels of the specific endothelial marker CD31 were similarly increased (Fig. 2C). Functional experiments indicated that a time-dependent increase in the diameter of neovascularization was observed as evidenced using a Matrigel angiogenesis assay (7 days, $P<0.01 ; 15$ days, $P<0.01$; Fig. 2D).

\section{MEG3 promotes the differentiation of ADSCs into vascular endothelial cells}

As shown in Fig. 3A, qPCR analysis revealed that the expression levels of MEG3 were markedly increased by $~ 70$-fold in response to differentiation signals in ADSCs. The results of subcellular fractionation analyses revealed that MEG3 was mainly distributed in the cytoplasm of ADSCs, suggesting their posttranscriptional regulatory potentials (Fig. 3B).

In the present study, MEG3 was further knocked down in the ADSCs to validate its effect on the differentiation of ADSCs. Notably, knockdown of MEG3 markedly decreased the mRNA and protein expression levels of endothelial marker genes, including CD31, vWF and Factor VIII ( $P<0.01$ vs. control group; Fig. 4A and B). Consistent with these results, immunofluorescence analysis demonstrated that the expression levels of CD31 were decreased in the ADSCs transfected with MEG3 shRNA (Fig. 4C). Functionally, the diameter measured from the neovascularization was significantly decreased in these cells ( $P<0.01$ vs. control group; Fig. 4D). In addition, overexpression of MEG3 significantly upregulated the protein expression levels of endothelial marker genes, including CD31, vWF and Factor VIII ( $P<0.001$ vs. control group; Fig. 5A). Functionally, the diameter measured from the neovascularization was significantly increased in these cells ( $P<0.001$ vs. control group; Fig. $5 B$ ). These results indicated that MEG3 could promote the differentiation of ADSCs into vascular endothelial cells.

miR-145-5p is a target of MEG3 in ADSCs: Given the essential role of MEG3 in the promotion of ADSC differentiation into vascular endothelial cells, it is of great interest to identify the target genes. Using target gene prediction online software (TargetScanHuman v7.2, http://www.targetscan.org/vert_72/), it was preliminarily predicted that miR-145-5p was the target gene of MEG3 (Fig. 6A) ${ }^{19}$, 20 . Luciferase reporter gene analysis revealed that miR-145-5p overexpression markedly decreased the luciferase activity of MEG3, while this inhibition was blocked by mutating the potential binding domains (Fig. 6B). Furthermore, RT-qPCR analysis revealed that the overexpression of MEG3 inhibited miR-145-5p expression (Fig. 6C). These data suggested that MEG3 directly sponged miR-145-5p to regulate the expression levels of miR-145-5p.

To further identify the target gene of miR-145-5p, KLF4 was further predicted as the possible candidate using TargetScanHuman based on the essential role of KLF4 in ADSC differentiation (Fig. 6D) ${ }^{21}$. Luciferase reporter gene analysis demonstrated that miR-145-5p overexpression markedly decreased the luciferase activity of the KLF4 3'-UTR region. By contrast, such an inhibitory effect was abrogated when the potential seed sequences were mutated in the 3'-UTR region of KLF4 (Fig. 6E). Additionally, western blotting revealed that miR-145-5p overexpression downregulated the protein expression levels of KLF4 in 
ADSCs (Fig. 6F). However, MEG3 overexpression upregulated the protein expression levels of KLF4 (Fig. 6G). These data suggested that miR-145-5p directly acted on the 3'-UTRs of KLF4 to regulate the expression levels of KLF4.

\section{MEG3 induces endothelial differentiation of ADSCs by targeting miR-145-5p/KLF4}

As shown in Fig. 7A, the present study examined the expression levels of miR-145-5p and KLF4 in response to ADSC differentiation and revealed that the expression levels of miR-145-5p were significantly decreased ( $P<0.01 ; 15$ vs. 0 days), while the mRNA expression levels of KLF4 were correspondingly increased ( $P<0.01 ; 15$ vs. 0 days). To determine the roles of miR-145-5p and KLF4 in mediating the function of MEG3 in ADSC differentiation, miR-145-5p and KLF4 were knocked down in ADSC cells transfected with MEG3 shRNA. It was demonstrated that the mRNA and protein expression levels of CD31, vWF and Factor VIII were markedly decreased in the cells transfected with MEG3 shRNA. By contrast, knockdown of miR-145-5p via its specific inhibitors partially antagonized such inhibitory effects of MEG3 shRNA. Notably, when KLF4 was further knocked down, such a neutralization by miR-145-5p inhibitors was reduced (Fig. 7B and C). Consistently, immunofluorescence analysis of CD31 expression confirmed these results (Fig. 7D). Functionally, Matrigel angiogenesis analysis revealed that miR-145-5p inhibitors enlarged the diameter of neovascularization in MEG3 shRNA-treated cells, while KLF4 shRNA recapitulated the effect of miR-145-5p inhibitors in these cells (Fig. 7E). These data indicated that MEG3 served its essential role in the regulation of ADSC differentiation via the miR-145-5p/KLF4 axis.

\section{Discussion}

ADSCs are derived from adipose mesenchymal tissue, and are also called adipose-derived mesenchymal stem cells. Therefore, ADSCs have the potential to differentiate into endothelial cells. Although a few studies on the directional differentiation of ADSCs into endothelium exist ${ }^{22}, 23$, to the best of our knowledge, the detailed mechanism via which ADSCs differentiate into endothelial cells remains unclear. Therefore, the purpose of the present study was to further illustrate such mechanisms.

In the present study, from the viewpoint of the extracellular matrix and microenvironment of the cells, the method of $\mathrm{FN}$ coating, EGM2-MV and $50 \mathrm{ng} / \mathrm{ml} \mathrm{VEGF}_{165}$ induction was used to induce the differentiation of vascular endothelial cells, as previously described ${ }^{24}$. After 15 days of endothelial induction, the expression levels of endothelial cell-specific markers ${ }^{25}$, including CD31, vWF and Factor VIII, were investigated in the ADSCs. The results demonstrated that the three markers were all expressed. In addition, the immunofluorescence assay demonstrated that endothelial cell-specific marker CD31 was positive. On the other hand, the Matrigel angiogenesis assay is a specific experiment to detect the function of endothelial cells ${ }^{26}$. Using three-dimensional Matrigel culture in vitro, the present study revealed that most of the induced ADSCs exhibited protuberances, which were connected with each other and connected completely, forming a large number of tubules connected by monolayer cells. The aforementioned results indicated that ADSCs were successfully induced and possessed the biological function of mature endothelial cells. 
MEG3 has become a research focus in the IncRNA field, and is involved in regulating the differentiation of stem cells $17,18,27$. In a recent study, investigators reported that IncRNA MEG3 could activate the VEGF signaling pathway and inhibited the Wnt/ $\beta$-catenin signaling pathway, thus inducing the angiogenesis of 3T3-L1 preadipocytes ${ }^{28}$. This finding consolidates the hypothesis that MEG3 is a a predicted related gene involved in the endothelial differentiation of ADSCs. In the present study, the expression levels of MEG3 were increased after 15 days of ADSC endothelial induction. Interestingly, the expression levels of endothelial cell-specific markers CD31, vWF and Factor VIII were all decreased in response to MEG3 knockdown. Consistently, the formation of tubules was also decreased in the MEG3-knockdown cells. These results suggested that MEG3 potentially promoted endothelial differentiation and upregulated the expression levels of endothelial cell-specific markers.

Given the essential role of miRNAs in mediating the function of IncRNAs, the present study screened out the potential effector of MEG3 using online tools and luciferase reporter assays. The present study revealed that miR-145-5p was the target gene of IncRNA MEG3. Notably, miR-145 can regulate microvascular growth and development by acting on the target gene Flil (Ets transcription factor Friend leukemia virus integration 1) ${ }^{29}$. In addition, miR-145 is abundant in primary cultured vascular cells but its expression is limited in dedifferentiated vascular cells. It has been well established that miR-145 serves an important role in phenotypic transformation of vascular cells and is an important regulator in the process of vascular cell differentiation ${ }^{30-32}$. The present data indicated that the expression levels of miR-145-5p were decreased after endothelial differentiation of ADSCs. Importantly, miR-145-5p inhibitor partially blocked the reduction of endothelial cell-specific markers caused by MEG3 knockdown, suggesting that miR-145-5p was a target miRNA of MEG3 and mediated its regulatory role in endothelial differentiation of ADSCs.

KLF4 has been demonstrated to be a critical regulator involved in endothelial cell inflammation, macrophage gene expression and smooth muscle cell proliferation and differentiation ${ }^{33-36}$. In recent years, several studies have also revealed the effects of KLF4 on endothelial differentiation. For example, Cantoni et al ${ }^{37}$ reported that human progenitor perivascular cells, which have the same immunophenotype as human ADSCs, exhibit remarkable vasculogenic potential via the upregulation of KLF4 expression. Windmolders et a/ ${ }^{38}$ reported that mesenchymal stem cells promote the migration of endogenous CSCs (Cardiac Atrial appendage Stem Cells) in the myocardium by releasing paracrine factors. The migrated cells, which possess high expression levels of KLF4, exhibit high acetaldehyde dehydrogenase activity and could differentiate towards cardiomyocytes. In addition, KLF4, which triggers phenotypic transformation of vascular smooth muscle cells ${ }^{39}$, promotes the proliferation and migration of vascular smooth muscle cells and downregulates the expression levels of smooth muscle cell-specific markers ${ }^{40}$. It should be noted that KLF4 is inhibited by miR-145 in human papillomavirus-positive cells, which leads to regulation of the life cycle of host cells ${ }^{41}$. In the present study, the expression levels of KLF4 were increased after endothelial induction of ADSCs. Furthermore, knockdown of KLF4 markedly antagonized the effects of miR-145-5p inhibitor on the endothelial transformation in ADSCs transfected 
with MEG3 shRNA. These results suggested that KLF4 per se functioned as an output gene of the MEG3/miR-145-5p axis.

In summary, the present study demonstrated that MEG3 served important roles in the differentiation of ADSCs into vascular endothelial cells. Mechanistically, it targeted and reduced the expression levels of miR-145-5p, which further upregulated the expression levels of KLF4, thus leading to the promotion of ADSC endothelial differentiation. The present findings provided novel insights into the mechanism involved in the endothelial differentiation of ADSCs.

\section{Declarations}

Ethics approval and consent to participate: All operations were approved by the Ethics Research Committee of the Heilongjiang Academy of Traditional Chinese Medicine (permit no. SY3R-2019004; Harbin, China).

Patient consent for publication: Not applicable.

Competing interests: The authors declare that they have no competing interests.

Availability of data and materials: All data generated or analysed during this study are included in this published article.

Authors' contributions: $\mathrm{HZ}$ conceived and designed the analysis. GL, BW and LY performed the experiments. $\mathrm{XM}$ and $\mathrm{XY}$ analyzed and interpreted the data. $\mathrm{XY}$ drafted the manuscript. $\mathrm{HZ}$ confirm the authenticity of all the raw data. All authors read and approved the final manuscript.

Funding: The present study was supported by the Traditional Chinese Medicine Research Project of Heilongjiang (grant no. ZHY2020-041 and ZHY19-062).

Acknowledgements: The authors would like to thank Nanjing Berke Biology Co., Ltd. provided technical support of adipose-derived stem cell separation technology.

\section{References}

1. Ko SH, Nauta A, Wong V et al (2011) The role of stem cells in cutaneous wound healing: what do we really know? Plast Reconstr Surg 127(Suppl 1):10S-20S

2. Brassard JA, Lutolf MP (2019) Engineering Stem Cell Self-organization to Build Better Organoids. Cell Stem Cell 24(6):860-876

3. Fraser JK, Wulur I, Alfonso Z et al (2006) Fat tissue: an underappreciated source of stem cells for biotechnology. Trends Biotechnol 24(4):150-154

4. Sivan U, Jayakumar K, Krishnan LK (2014) Constitution of fibrin-based niche for in vitro differentiation of adipose-derived mesenchymal stem cells to keratinocytes. Biores Open Access 
$3(6): 339-347$

5. Hu R, Ling W, Xu W et al (2014) Fibroblast-like cells differentiated from adipose-derived mesenchymal stem cells for vocal fold wound healing. PLoS ONE 9(3):e92676

6. Mazini L, Rochette L, Amine M et al (2019) Regenerative Capacity of Adipose Derived Stem Cells (ADSCs), Comparison with Mesenchymal Stem Cells (MSCs).Int J Mol Sci, 20(10)

7. Ebrahimian TG, Pouzoulet F, Squiban $C$ et al (2009) Cell therapy based on adipose tissue-derived stromal cells promotes physiological and pathological wound healing. Arterioscler Thromb Vasc Biol 29(4):503-510

8. Atalay S, Coruh A, Deniz K (2014) Stromal vascular fraction improves deep partial thickness burn wound healing. Burns 40(7):1375-1383

9. Flynn RA, Chang HY (2014) Long noncoding RNAs in cell-fate programming and reprogramming. Cell Stem Cell 14(6):752-761

10. Mishra S, Verma SS, Rai V et al (2019) Long non-coding RNAs are emerging targets of phytochemicals for cancer and other chronic diseases. Cell Mol Life Sci 76(10):1947-1966

11. Dong P, Xiong Y, Yue J et al (2019) Exploring IncRNA-Mediated Regulatory Networks in Endometrial Cancer Cells and the Tumor Microenvironment: Advances and Challenges.Cancers (Basel), 11(2)

12. Brown WM (2015) Exercise-associated DNA methylation change in skeletal muscle and the importance of imprinted genes: a bioinformatics meta-analysis. Br J Sports Med 49(24):1567-1578

13. Kumar MM, Goyal R (2017) LncRNA as a Therapeutic Target for Angiogenesis. Curr Top Med Chem 17(15):1750-1757

14. Piccoli MT, Gupta SK, Viereck J et al (2017) Inhibition of the Cardiac Fibroblast-Enriched IncRNA Meg3 Prevents Cardiac Fibrosis and Diastolic Dysfunction. Circ Res 121(5):575-583

15. Sun X, Luo LH, Feng L et al (2018) Down-regulation of IncRNA MEG3 promotes endothelial differentiation of bone marrow derived mesenchymal stem cells in repairing erectile dysfunction. Life Sci 208:246-252

16. Zhuang W, Ge X, Yang S et al (2015) Upregulation of IncRNA MEG3 Promotes Osteogenic Differentiation of Mesenchymal Stem Cells From Multiple Myeloma Patients By Targeting BMP4 Transcription. Stem Cells 33(6):1985-1997

17. You D, Yang $C$, Huang J et al (2019) Long non-coding RNA MEG3 inhibits chondrogenic differentiation of synovium-derived mesenchymal stem cells by epigenetically inhibiting TRIB2 via methyltransferase EZH2. Cell Signal 63:109379

18. Li Z, Jin C, Chen S et al (2017) Long non-coding RNA MEG3 inhibits adipogenesis and promotes osteogenesis of human adipose-derived mesenchymal stem cells via miR-140-5p. Mol Cell Biochem 433(1-2):51-60

19. Yu L, Qu H, Yu Y et al (2018) LncRNA-PCAT1 targeting miR-145-5p promotes TLR4-associated osteogenic differentiation of adipose-derived stem cells. J Cell Mol Med 22(12):6134-6147 
20. Zhou T, Chen S, Mao X (2019) miR-145-5p affects the differentiation of gastric cancer by targeting KLF5 directly. J Cell Physiol 234(5):7634-7644

21. Zhang J, Liu H, Lin H et al (2017) Sp1 is a competitive endogenous RNA of KIf4 during odontoblast differentiation. Int J Biochem Cell Biol 85:159-165

22. Tseng YC, Roan JN, Ho YC et al (2017) An in vivo study on endothelialized vascular grafts produced by autologous biotubes and adipose stem cells (ADSCs). J Mater Sci Mater Med 28(10):166

23. Yang Y, Cai Y, Zhang Y et al (2018) Exosomes Secreted by Adipose-Derived Stem Cells Contribute to Angiogenesis of Brain Microvascular Endothelial Cells Following Oxygen-Glucose Deprivation In Vitro Through MicroRNA-181b/TRPM7 Axis. J Mol Neurosci 65(1):74-83

24. Bento LW, Zhang Z, Imai A et al (2013) Endothelial differentiation of SHED requires MEK1/ERK signaling. J Dent Res 92(1):51-57

25. Harmsen MJ, Wong CFC, Mijatovic V et al (2019) Role of angiogenesis in adenomyosis-associated abnormal uterine bleeding and subfertility: a systematic review. Hum Reprod Update 25(5):647-671

26. Cao Y, Sun Z, Liao L et al (2005) Human adipose tissue-derived stem cells differentiate into endothelial cells in vitro and improve postnatal neovascularization in vivo. Biochem Biophys Res Commun 332(2):370-379

27. Sommerkamp P, Renders S, Ladel L et al (2019) The long non-coding RNA Meg3 is dispensable for hematopoietic stem cells. Sci Rep 9(1):2110

28. Huang X, Fu C, Liu W et al (2019) Chemerin-induced angiogenesis and adipogenesis in 3T3-L1 preadipocytes is mediated by IncRNA Meg3 through regulating Dickkopf-3 by sponging miR-217. Toxicol Appl Pharmacol 385:114815

29. Larsson E, Fredlund Fuchs P, Heldin J et al (2009) Discovery of microvascular miRNAs using public gene expression data: miR-145 is expressed in pericytes and is a regulator of Fli1. Genome Med 1(11):108

30. Lin X, Yang F, Qi X et al (2019) LncRNA DANCR promotes tumor growth and angiogenesis in ovarian cancer through direct targeting of miR-145. Mol Carcinog 58(12):2286-2296

31. Chandy M, Ishida M, Shikatani EA et al (2018) c-Myb regulates transcriptional activation of miR143/145 in vascular smooth muscle cells. PLoS ONE 13(8):e0202778

32. Cordes KR, Sheehy NT, White MP et al (2009) miR-145 and miR-143 regulate smooth muscle cell fate and plasticity. Nature 460(7256):705-710

33. Hamik A, Lin Z, Kumar A et al (2007) Kruppel-like factor 4 regulates endothelial inflammation. J Biol Chem 282(18):13769-13779

34. Feinberg MW, Cao Z, Wara AK et al (2005) Kruppel-like factor 4 is a mediator of proinflammatory signaling in macrophages. J Biol Chem 280(46):38247-38258

35. Feinberg MW, Lin Z, Fisch S et al (2004) An emerging role for Kruppel-like factors in vascular biology. Trends Cardiovasc Med 14(6):241-246 
36. Yan FF, Liu YF, Liu Y et al (2008) KLF4: a novel target for the treatment of atherosclerosis. Med Hypotheses 70(4):845-847

37. Cantoni S, Bianchi F, Galletti M et al (2015) Occurring of In Vitro Functional Vasculogenic Pericytes from Human Circulating Early Endothelial Precursor Cell Culture. Stem Cells Int 2015:943671

38. Windmolders S, De Boeck A, Koninckx R et al (2014) Mesenchymal stem cell secreted platelet derived growth factor exerts a pro-migratory effect on resident Cardiac Atrial appendage Stem Cells. J Mol Cell Cardiol 66:177-188

39. Yoshida T, Kaestner KH, Owens GK (2008) Conditional deletion of Kruppel-like factor 4 delays downregulation of smooth muscle cell differentiation markers but accelerates neointimal formation following vascular injury. Circ Res 102(12):1548-1557

40. Davis-Dusenbery BN, Chan MC, Reno KE et al (2011) down-regulation of Kruppel-like factor-4 (KLF4) by microRNA-143/145 is critical for modulation of vascular smooth muscle cell phenotype by transforming growth factor-beta and bone morphogenetic protein 4. J Biol Chem 286(32):2809728110

41. Gunasekharan V, Laimins LA (2013) Human papillomaviruses modulate microRNA 145 expression to directly control genome amplification. J Virol 87(10):6037-6043

\section{Figures}

\section{Figure 1}

Characterization of ADSCs. ADSCs were isolated from the adipose tissues. At the third passage, the expression levels of ADSCs markers (A) CD44 and CD90, and (B) CD31 and HLA-DR were analyzed via flow cytometry. ADSCs, adipose-derived stem cells; HLA-DR, major histocompatibility complex, class II antigen-associated.

\section{Figure 2}

Identification of endothelial cells induced by ADSCs. The third-generation ADSCs were induced for the indicated times, and samples were collected and (A) reverse transcription-quantitative PCR and (B) western blotting were used to examine endothelial markers, including CD31, vWF and Factor VIII. (C) Immunofluorescence analysis of CD31 expression, $200 \times$. (D) Matrigel angiogenesis assays, $200 \times$. Statistical data are presented as the mean \pm SD. $n=3$. ${ }^{*} P<0.05$, ${ }^{* *} P<0.01$ vs. control group. The experiments were independently repeated three times. ADSCs, adipose-derived stem cells; d, days; vWF, von Willebrand factor. 


\section{Figure 3}

Effects of MEG3 on endothelial differentiation of adipose-derived stem cells. (A) On days 0 and 15 after endothelial induction, the expression levels of long non-coding RNA MEG3 were detected via reverse transcription-quantitative PCR. ${ }^{* *} \mathrm{P}<0.01$ vs. 0 days group. (B) Expression levels of MEG3 in nuclear and cytoplasmic fractions were measured via reverse transcription-quantitative PCR. U6 and GAPDH were used as a positive control for the nuclear and cytoplasmic fractions, respectively. Statistical data are presented as the mean \pm SD. The experiments were independently repeated three times. $d$, days; MEG3, maternally expressed 3 .

\section{Figure 4}

Effects of MEG3 knockdown on endothelial differentiation of ADSCs. After treatment with sh-NC and shMEG3, ADSCs were collected and (A) reverse transcription-quantitative PCR and (B) western blotting were used to examine endothelial markers, including CD31, vWF and Factor VIII. (C) Immunofluorescence analysis of CD31 expression, $200 \times$. (D) Matrigel angiogenesis assay, $200 \times$. Statistical data are presented as the mean $\pm S D . n=3 .{ }^{* *} P<0.01$ vs. control group. The experiments were independently repeated three times. ADSCs, adipose-derived stem cells; MEG3, maternally expressed 3; NC, negative control; sh, short hairpin RNA; vWF, von Willebrand factor.

\section{Figure 5}

Effects of MEG3 overexpression on endothelial differentiation of ADSCs. (A) After treatment with pc-NC and pc-MEG3, ADSCs were collected and western blotting was used to detect endothelial markers, including CD31, vWF and Factor VIII. (B) Matrigel angiogenesis assay. Scale bar, $200 \mu \mathrm{m}$. Statistical data are presented as the mean $\pm \mathrm{SD} . \mathrm{n}=3 .{ }^{\star \star \star} \mathrm{P}<0.001$ vs. control group. The experiments were independently repeated three times. ADSCs, adipose-derived stem cells; MEG3, maternally expressed 3; NC, negative control; pc, pcDNA3.1; vWF, von Willebrand factor.

\section{Figure 6}

miR-145-5p is a target of long non-coding RNA MEG3 in adipose-derived stem cells. (A) Binding sites and mutations between miR-145-5p and MEG3. (B) Dual-luciferase reporter assay of the interaction between miR-145-5p and MEG3. (C) After treatment with pc-MEG3, the expression levels of miR-145-5p were detected by reverse transcription-quantitative PCR. (D) Binding sites and mutations between miR-145-5p 
and KLF4. (E) Dual-luciferase reporter assay of the interaction between miR-145-5p and KLF4. (F) After treatment with miR-145-5p mimic, the expression levels of KLF4 were detected via western blotting. (G) After treatment with pc-MEG3, the expression levels of KLF4 were detected by western blotting. Statistical data are presented as the mean $\pm \mathrm{SD} . \mathrm{n}=3 .{ }^{* \star} \mathrm{P}<0.01,{ }^{* \star \star} \mathrm{P}<0.001$ vs. control group. The experiments were independently repeated three times. KLF4, Krüppel like factor 4; MEG3, maternally expressed 3; miR-1455p, microRNA-145-5p; Mut, mutant; NC, negative control; pc, pcDNA3.1; WT, wild-type.

\section{Figure 7}

MEG3/miR-145-5p/KLF4 regulates the differentiation of ADSCs into vascular endothelial cells. (A) After endothelial induction of ADSCs, the mRNA expression levels of miR-145-5p and KLF4 were detected by reverse transcription-quantitative PCR. Cells were transfected with MEG3 shRNA in combination with or without miR-145-5p inhibitors and KLF4 shRNA. (B) Reverse transcription-quantitative PCR and (C) western blotting were used to detect endothelial markers, including CD31, vWF and Factor VIII. (D) Immunofluorescence analysis of CD31 expression, $200 \times$. (E) Matrigel angiogenesis assay, $200 \times$.

Statistical data are presented as the mean $\pm S D$. $n=3$. ${ }^{* *} P<0.01$ vs. control group. ${ }^{\# \#} P<0.01$ vs. sh-MEG3 + $N C$ inhibitor group. ${ }^{++} \mathrm{P}<0.01$ vs. sh-MEG3 + miR-145-5p inhibitor group. The experiments were independently repeated three times. ADSCs, adipose-derived stem cells; KLF4, Kruppel like factor 4; MEG3, maternally expressed 3; miR-145-5p, microRNA-145-5p; NC, negative control; shRNA/sh, short hairpin RNA; vWF, von Willebrand factor.

\section{Supplementary Files}

This is a list of supplementary files associated with this preprint. Click to download.

- S1.tif 